\title{
Multi-timescale measurements of brain responses in visual cortex during functional stimulation using time-resolved spectroscopy
}

\author{
Solomiya Lebid*, Raymond O’Neill, Charles Markham, Tomás Ward, and Shirley Coyle \\ Biomedical Research Group, National University of Ireland, Maynooth, Co. Kildare, Ireland
}

\begin{abstract}
Studies of neurovascular coupling (hemodynamic changes and neuronal activation) in the visual cortex using a timedomain single photon counting system have been undertaken. The system operates in near infrared (NIR) range of spectrum and allows functional brain monitoring to be done non-invasively. The detection system employs a photomultiplier and multi-channel scaler to detect and record emerging photons with sub-microsecond resolution (the effective collection time per curve point is $~ 200 \mathrm{~ns}$ ). Localisation of the visual evoked potentials in the brain was done using knowledge obtained from electroencephalographic (EEG) studies and previous frequency-domain optical NIR spectroscopic systems. The well-known approach of visual stimulation of the human brain, which consists of an alternating black and white checkerboard pattern used previously for the EEG study of neural responses, is applied here. The checkerboard pattern is synchronized with the multi-channel scaler system and allows the analysis of time variation in back-scattered light, at different stimulation frequencies. Slow hemodynamic changes in the human brain due to $\mathrm{Hb}-$ $\mathrm{HbO}_{2}$ changes in the blood flow were observed, which is evidence of the system's capability to monitor these changes. Monocular visual tests were undertaken and compared with those done with an EEG system. In some subjects a fast optical response on a time scale commensurate with the neural activity associated with the visual cortex was detected. Future work will concentrate on improved experimental protocols and apparatus to confirm the existence of this important physiological signal.
\end{abstract}

Keywords: single photon counting, time-domain system, neurovascular signal, neuronal activation

\section{INTRODUCTION}

The fact that at least third of the cerebral cortex is devoted to visual processing makes the sense of vision one of the most important human senses. Therefore, it is not surprising that a large number of interdisciplinary investigations in neuropsychology are dominated by visual studies using various techniques [1-9]. On the other hand, the evolution of instrumentation for neuropsychological studies is a result of the contribution from many various disciplines. It allows better understanding of human brain function on many levels. These techniques could be divided along invasive and noninvasive lines. Current non-invasive multi-timescale functional brain analysis gives hope that tests done presently invasively will be replaced in the near future [10-12]. Therefore, in this work, two non-invasive measurement techniques for visual stimulation studies in human adults are described. This study continues research done previously [13,14] on the development of an alternative optical system for acquisition of multi timescale evoked potentials in the human brain and the main outline of the current work is done in a comparative study to obtain those signals on ms-timescales. Before descriptions of the experimental methods and results are presented, a brief introduction to the physiology of the human visual processing system is given along with a background on previously known work for its analysis.

\subsection{Visual perception}

Schematically, organisation of the visual system consists of the hierarchical processing of the information starting from eyes and up to the visual cortex of the human brain. It is the eye that first senses visual stimulus, e.g. checkerboard pattern (see Figure 1). It consists of the retina which itself contains some cells called retinal ganglion cells. The information from these cells enters into the optic nerve which consists of $\sim 1$ million fibres. Approximately half the fibres (an optic nerve of each eye) cross in the optic chiasm which marks the transition from the optic nerve to the optic tract. The effect of this crossing is that axons carrying information from the right and left parts of the two retinas travel together on the contralateral side of the brain [2]. So, when we accept external information we accept it from two visual

* solomiya.lebid@eeng.nuim.ie; phone 3531 7086381; fax 3531 7086247; biomed.eeng.nuim.ie 
fields - the right and the left. Fibres that exit the optic tract enter the lateral geniculate nucleus (LGN) of the thalamus (see Figure 1). After that information transmitted via fibres travels different paths to the midbrain delivering information on its way there to other parts of the brain $[1,2,15]$.

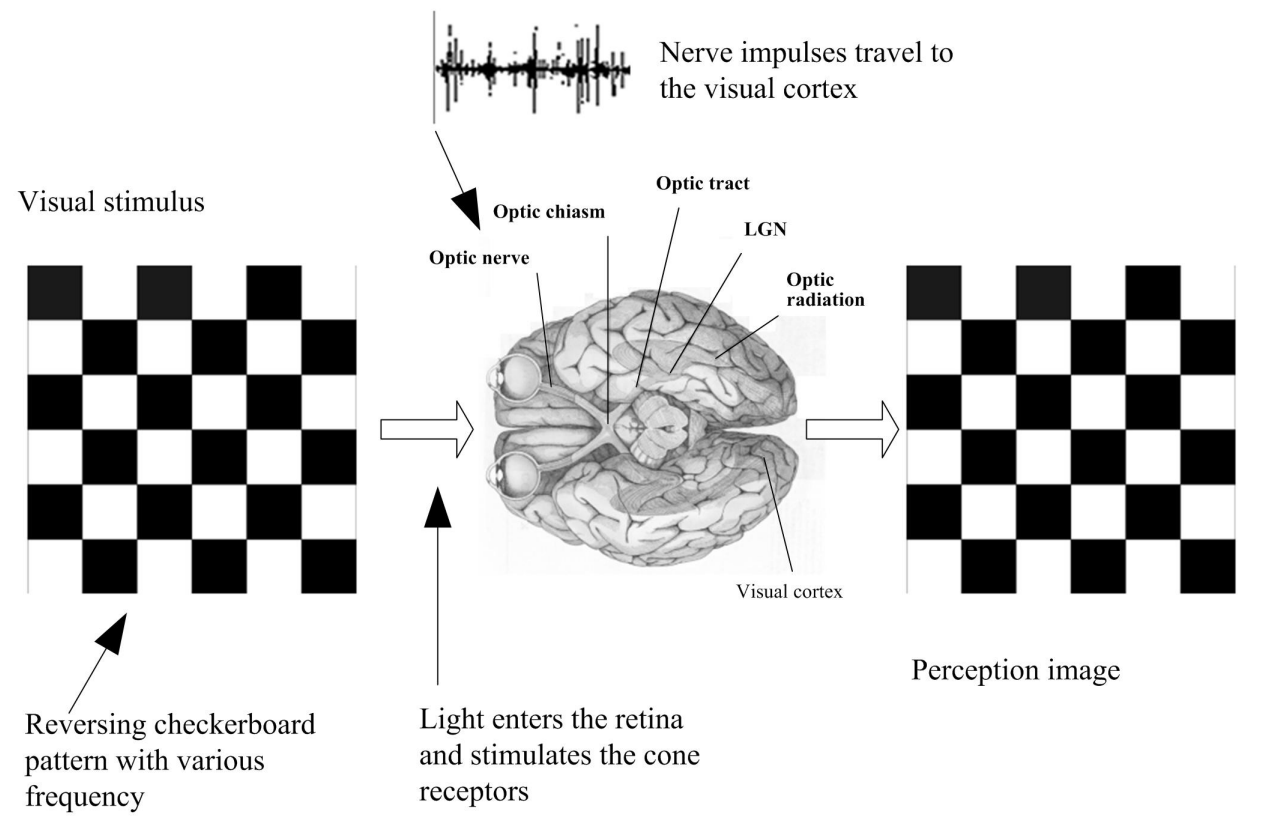

Figure 1: Visual stimulation of the brain: Schematic representation of the information processing from stimulus to perception of the image.

The retinal ganglion cells synapse in the LGN and the next set of neurons carry information to the visual cortex through the cerebral white matter called the optic radiation. The largest part of the information goes to the primary visual cortex, also known as area VI or the striate cortex, located in the occipital lobe. The visual cortex receives a virtual image of the real image. Other areas (V2, V3, V4, V5) receive connections from further cortical areas. Such techniques as single-cell microelectrode recording and nerve fibre tracing have allowed investigation of these areas [7,8], but most of the existing experimental techniques cannot be applied for the study of human visual cortex from the ethical point of view [2]. In any case, signal processing in a human brain is more complicated than that performed by any artificial system.

\subsection{Existing techniques for functional brain analysis}

As it was mentioned before, a large number of various disciplines have made their contribution to the current variety of brain study techniques, allowing analysis of brain activity on various levels. Some of them are worth mentioning here despite their clinic-only application. The general principle of their operation is through measuring intrinsic activityrelated changes in tissue (such as hemodynamics) which arise due to functional physiological changes. The slow hemodynamic changes occur within seconds after the stimulus is activated and are based on the increased blood perfusion in an area of activated neurons due to neurovascular coupling. Stimulus increases oxygen consumption by active neurons which results in an increase in the blood flow. Changes in hemoglobin concentration and oxygenation resulting from this interaction can be measured using techniques, such as positron emission tomography (PET), functional magnetic resonance imaging (fMRI) and in vivo near-infrared spectroscopy (ivNIRS). Positron emission tomography $(P E T)$, first allowed imaging of the blood flow caused by enhanced neural activity during cognition. It measures brain oxygen consumption, blood flow and glucose metabolism. Blood flow appears to be the most reliable measurand. To measure glucose metabolism, radioactive glucose is injected into the subject and is consumed by metabolically active cells. The radioactive particles emit positrons. When positrons are emitted and collide with electrons, they form gamma-rays which exit the head and are detected. Areas with higher metabolic activity will take 
more glucose and produce more gamma rays. Blood flow can be measured by injecting radioactive water (hydrogen and oxygen 15) into the subject. The main disadvantages of this technique are that it invasive and has a poor temporal resolution [1, 2]. Functional magnetic resonance imaging (fMRI) is based on the changes of the magnetic properties of atoms. This system can detect protons which respond in the magnetic field. When radiowaves are then passed through the head, the reverberations produced by the resonance of hydrogen molecules are detected by the scanner. The concentration of oxygen in blood can affect the magnetic properties of haemoglobin. Therefore, this allows fMRI to analyse concentration of oxygen in blood and blood flow by picking up these functionally induced changes. fMRI is worth mentioning because it is non-invasive, but on the other hand, its effectiveness decreases in venous oxygen content, has poor temporal resolution, and is sensitive to movement of the subject. In addition its size makes it unsuitable for nonclinical applications. Summarising, it can be underlined that these techniques offer information about functional brain activity but at the expense of complicated measurement, spatial or temporal resolution losses, signal aetiology, practicality and cost, but there is no existing system that meets all requirements. There are two features which have critical importance in this current study: the technique must be non-invasive, possess a high time-resolution and be suitable for non-clinical applications. Therefore, only two systems will be applied in current work: electroencephalography (EEG) and NIRS.

In vivo near-infrared spectroscopy ( $i v \mathrm{NIRS}$ ) is a rapidly expanding technique that has found applications outside the clinic. The interrogation of biological tissue with near-infrared light $(700-1300 \mathrm{~nm})$ allows non-invasive evaluation of important brain metabolic processes such as tissue oxygenation, haemoglobin content, and cytochrome oxydase $\left(\mathrm{CytO}_{2}\right)$ $[2,5,9,13,16]$. A number of studies undertaken by leading laboratories have shown that it is possible to measure brain responses during motor, cognitive, and visual stimulation [9, 14, 17, 18, 19]. The absorption of light by deoxyhaemoglobin $(\mathrm{Hb})$ gives a peak at $\sim 760 \mathrm{~nm}$ and that of oxy-haemoglobin $\left(\mathrm{HbO}_{2}\right)$ peaks at $900 \mathrm{~nm}$. At $\sim 800 \mathrm{~nm}$ the attenuation of $\mathrm{Hb}$ is the same as $\mathrm{HbO}_{2}$ and therefore, the total amount of haemoglobin $\mathrm{Hb}_{\text {total }}=\mathrm{Hb}+\mathrm{HbO}_{2}$ can be assessed. If attenuation of light is determined at $\sim 760 \mathrm{~nm}$, mainly $\mathrm{Hb}$ is evaluated. $\mathrm{CytO}_{2}$ an import brain metabolic enzyme also displays absorption from $\sim 780 \mathrm{~nm}$ to $\sim 870 \mathrm{~nm}$. The $\mathrm{CytO}_{2}$ absorption peak is larger than that displayed by $\mathrm{Hb}$ and in contrast to $\mathrm{Hb}, \mathrm{CytO}_{2}$ content is stable over days and therefore, it is easier to detect it with one wavelength only. However, the total amount of absorption of $\mathrm{Hb}$ and $\mathrm{HbO}_{2}$ is larger than that of $\mathrm{CytO}_{2}$. Investigations done in vivo using NIRS has concentrated on using frequency domain techniques to obtain information on the optical path-length of propagated photon through the living tissue, which is the highly scattering medium. However, with this approach, currently achieved best value for the sampling rate is $\sim 200 \mathrm{~Hz}$ and the technique is very expensive. On the "slow" time scale NIRS has proved its ability to record hemodynamic changes in the human brain $[3,9,10,13,16,18]$, however on the "fast" timescale which is important for neural event detection it is only just beginning to make progress $[11,12,14$, 20]. The previously described slow biochemical changes in the human brain $(\sim 10 \mathrm{~s})$ are indirectly associated with neuronal firing. Recently achieved experimental evidence for the much smaller neuronal activity signal (NAS) on the time-scale approximately of $100 \mathrm{~ms}$, requires the development of special apparatus for its acquisition [12, 14, 20, 21, 22, 23]. It has been noticed by Gratton et al. that during a visual stimulation task, evoked-responses in the brain measured by frequency-domain NIRS system showed systematic errors appearing as a function of the eccentricity of the vertical black and white bars. The short latency of these errors suggested that it is connected with some effects reflecting the activity of neurons [23]. The terms optical evoked responses (OER) or more accurately the fast optical evoked response have been used to refer to these signals.

The second technique used in this study is electroencephalography or EEG. The advantages of the EEG is that it is noninvasive for studies on humans, it has excellent temporal resolution, its easy to operate and can be used to measure the brain's response to a number of psychological variables. The electrical activity of the brain is recorded by placing electrodes over the human scalp according to the agreed 10-20 International Placement System [24], or they could be fitted into ant electrode cap. The acquired EEG signal is generated by the postsynaptic dendrites of millions of brain cells. The signal is very small and, therefore, has to be amplified and averaged if stimulus locked aspects of the EEG are to be acquired.

The ease of use of both NIRS and EEG makes them ideal for non-invasive research studies although problems connected with the positioning of optodes and electrodes $[16,19]$ as well as an incomplete knowledge on how the light propagates through the human head, which makes it difficult to calibrate the system, still remain. Previously undertaken studies on correlation between an optode distance and the depth of the brain area penetrated by light are shown that the depth on which a light penetrates tissue is approximately half of the distance between source and detector optodes [Gra00]. 
Variations of the source-detector distance were used to estimate the depth on which the evoked-response in the brain is the largest. These same studies have also shown that the obtained data becomes less reliable with an increase in the source-detector distance which could be explained by a decrease in photons with the increase of penetration depth. Nevertheless, it was proved that cerebrospinal fluid contained in the subarachnoid space between the superficial skull layer and deeper brain areas is not as much of an obstacle for propagating photons as it was reported before [25]. Evidence for that is the relationship between source-detector distance and penetration depth which is fairly predictable according to [21]. On the other hand, a change in scatter of the tissue will change the pathlength of the propagated photons and also alter the intensity of the measured signal. Nevertheless, changes in the signal due to scatter (neuron firing) are tiny in comparison to changes introduced by absorption (hemodynamic changes). The group of Obrig et al. performed a Monte-Carlo simulation based on the reported before knowledge of changes measured invasively on animals [22] and the multi-layer model of the human head [25]. According to this simulation, the magnitude of NAS is so small that the noise level and other influences in tissue are greater than the signal itself. The OERs generate only a tiny fraction of the $i v$ NIRS data signal $\Delta \mathrm{I} / \mathrm{I}=0.05 \%$ certainly, as it was proved by experimental non-invasive measurement [20], with the remainder generated by noise processes of various sorts: muscular activation, respiration, heartbeat, shot noise, neuronal activity not of interest to the study. On the other hand, NIRS may offer the possibility of simultaneous and noninvasive measurement of neuronal and vascular signals in the brain with temporal resolution up to $1 \mathrm{~ms}$ [12, 20, 22]. According to Wolf et al., the measurement could be successfully realised using an adaptive filter for arterial pulsation removal using ahighly effective digital high pass filter and a crosscorrelation function between the optical data and the stimulation signal. This helped to filter out the tiny fraction of the signal of interest, which it was believed to be the NAS. Frequency-domain systems were used in the above mentioned studies. These systems use sinusoidally intensitymodulated light sources and rely on the phase shift of the modulation wave between incident and reflected signal to estimate time of flight of the photon through the tissue. A similar strategy is being investigated by the research group, LANL/USA [22]. This involves tomographic reconstruction using first light, or more generally using the impulseresponse measured with a temporal precision of $10^{-11} \mathrm{~s}$, this is a promising technique although signal-to-noise levels are an issue and the technique is expensive. Therefore, there is a need for a specialised system for such neurophysiololgical studies that can operate on multiple time-scales.

\section{EQUIPMENT AND METHODOLOGY}

A single photon counting system described in detail elsewhere [13, 14] makes it possible to record multi-timescale changes in the human brain with high resolution. Electroencephalography was applied to pick up brain neural activity in parallel and to prove that the chosen protocol did actually evoke some visual evoked potentials (VEP). On the other hand, neural activity in the brain is directly connected with the cerebral blood flow (CBF) changes as many laboratories have proved [5-7]. It was therefore, very important to make a comparatory measurement.

\subsection{Electroencephalography (EEG)}

EEG helps better understand neural activity changes during visual stimulation task. Event-related potentials (ERPs) are stimulus-locked transient features in the EEG. Usually the signal recorded with EEG could be very noisy. Therefore, it is useful to sum and average the signal representing a brain response to each stimulus. Repeating availability of stimulus in each recorded signal will make a resulting presentation very clear. Usually, a wave starts with a negative peak and welldefined latency with a positive peak afterwards. Therefore, they got names like N100, P200, and N300 which describe their polarity and latency. Such early waves, like N100 and P200 are the result of the perception of sensory stimulus. Later waves, like P300, are thought to represent a brain processing corresponding to decision-making, cognitive processing. Appearance of these later waves could be predicted under some definite circumstances but explanation of neural mechanisms causing it is currently not known [1].

\subsection{Photon counting system}

A high time-resolution photon counting system for extracting both slow and fast optical responses provides a very direct means of localising brain activity. It complements EEG studies as the tool for cheaply and non-invasively probing brain function. This time-resolved time-domain technique acquires the run time distribution of the photons passing through the tissue. In other words, it measures number of photons that passed through the tissue and entered the detector. It uses constant light sources: CW laser diodes with wavelengths $780 \mathrm{~nm}$ and $820 \mathrm{~nm}$, and optical power appr. $5 \mathrm{~mW}$. $800 \mathrm{MHz}$ 
gated photon counter/multiscaler was used for data acquisition. It contains ultra fast discriminators for the counting and gating inputs, two fast 32 bit counters, memory for storing the results, the timing and control logic and the PC bus interface. Photodetector, comprising photomultiplier tube with S20 photocathode for high IR response and a high-speed amplifier-discriminator was applied. An analogue acquisition is usually done by using lowpass filter, a photon counting is done by counting the detector pulses within subsequent time intervals by counter/timer combination. The difference consists in insensitivity of photon counting to the low frequency noise. There is also no baseline drift due to spurious currents in the PMT or in the PMT voltage divider [26]. In addition to these advantages, time-resolved technique improves localisation and characterisation of inhomogenities of the living tissue. A bifurcated fibre optic patch cord and multimode fibre optic patch cord are used as optodes for light delivery from the laser diode to the subject and for light delivery from the subject to the photodetector. Optodes were positioned in the area of visual cortex using a rubber band allowing flexibility in changing the position.

\section{PROTOCOLS}

The stimulus for the visual evoked-response is the reversing $6 \times 6$ checkerboard pattern displayed on the LCD monitor. A simple program was generating checkerboard pattern and driving the reversals of white and black squares periodically at various frequencies $(6-10 \mathrm{~Hz})$. In the middle of the pattern was placed a red circle as a focusing point. Full-field stimulation was done, which means simultaneous stimulation of the left and right visual fields or in other words, complete pattern. There were various protocols for acquiring slow and fast changes in the human brain developed.

\subsection{Hemodynamic changes in the brain: binocular and monocular study}

The C program generating black and white checkerboard pattern was synchronised with multichannel scaler in the way that data during checkerboard reversals were recorded into channel A and data during rest periods were recorded into channel B. The C-program was developed to generate reversals with various frequencies during fixed period of time and after that held the same rest period. Data obtained with a single-photon counting system were analysed off-line. First the data were filtered with the Butterworth low-pass filter. Finally, the data were averaged to reduce noise.

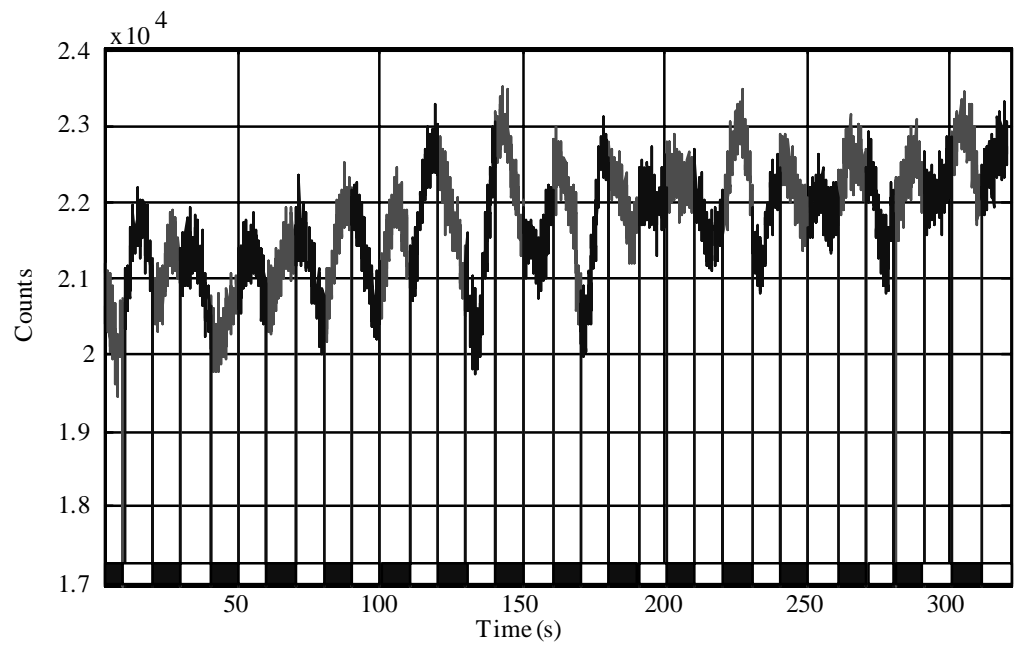

Figure 2: Hemodynamic response in the area of visual cortex of the human subject: Black squares in the bottom of the graph correspond to the reversals of the stimulus with frequency $8 \mathrm{~Hz}$, white squares correspond to the rest period.

First protocol was designed to record slow hemodynamic response in the visual cortex. Measurement was set-up on one wavelength only, $780 \mathrm{~nm}$, which give us information only about absorption of $\mathrm{Hb}$. Area of the visual cortex affected by visual stimulation on the longer time-scales, is larger than that affected by neuron firing and therefore, easier to localise. Optodes were placed on the subject's head in the area of visual cortex in the way that detector was placed approximately $2 \mathrm{~cm}$ above the inion and source optode to the left side of the detector. Distance between laser diode-optode and PMToptode was chosen to be $36.48 \mathrm{~mm}$ to penetrate $\sim 24 \mathrm{~mm}$ into the scull. The subject was placed in the dark room, approximately $1 \mathrm{~m}$ before the monitor where the checkerboard pattern was displayed. The C-program reversed the 
checkerboard at frequencies from 6 to $10 \mathrm{~Hz}$ during $10 \mathrm{~s}$ and after that held $10 \mathrm{~s}$ rest period. The whole session with one frequency took $320 \mathrm{~s}$. Optical signal was recorded with sampling frequency of $25 \mathrm{~Hz}$. Figure 2 represents response recorded from the visual cortex. Black squares on the bottom of the graph correspond to the reversals of the stimulus with frequency $8 \mathrm{~Hz}$, white squares correspond to the rest period.
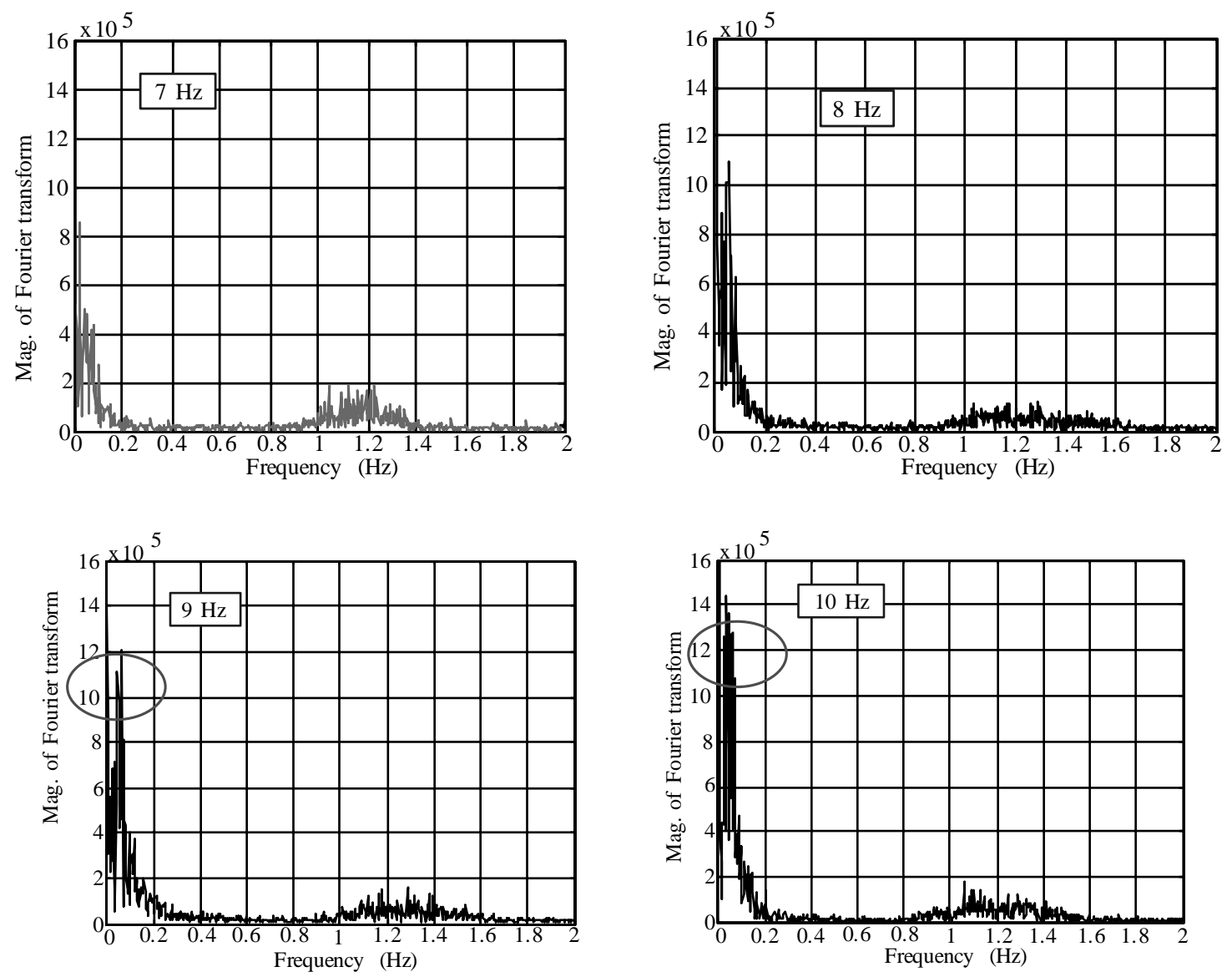

Figure 3: Fourier analysis of data obtained during tests performed with different frequencies of pattern reversal 7-10 Hz.

Obtained data were analysed further off-line. First, the data were low-pass filtered using the $2^{\text {nd }}$ order Butterworth filter with a $2 \mathrm{~Hz}$ cutoff. Afterwards, Fourier analysis (the fast Fourier transform (FFT)) was done (see Figure 3).

Figure 3 shows that the higher the frequency of the checkerboard reversal the higher the response on the lower frequencies. This corresponds to the slow signal or hemodynamic changes within the brain during the stimulation task. It is however true that on the lower frequencies there are also peaks at approximately $0.3 \mathrm{~Hz}$ that correspond to the respiration and at approximately $0.12 \mathrm{~Hz}$ that correspond to the Meyer wave [27, 28], but they are not always present in the optical signal (see Figure 4). Nevertheless, in the obtained data, the expected signal due to reversing stimulus at the frequency of $0.05-0.1 \mathrm{~Hz}$ is increasing obviously with the higher frequency of stimulus. Frequencies between $1 \mathrm{~Hz}$ and $2 \mathrm{~Hz}$ in Figure 3 are the heart rate or arterial pulsations in the visual cortex and their harmonics picked up by the light. 

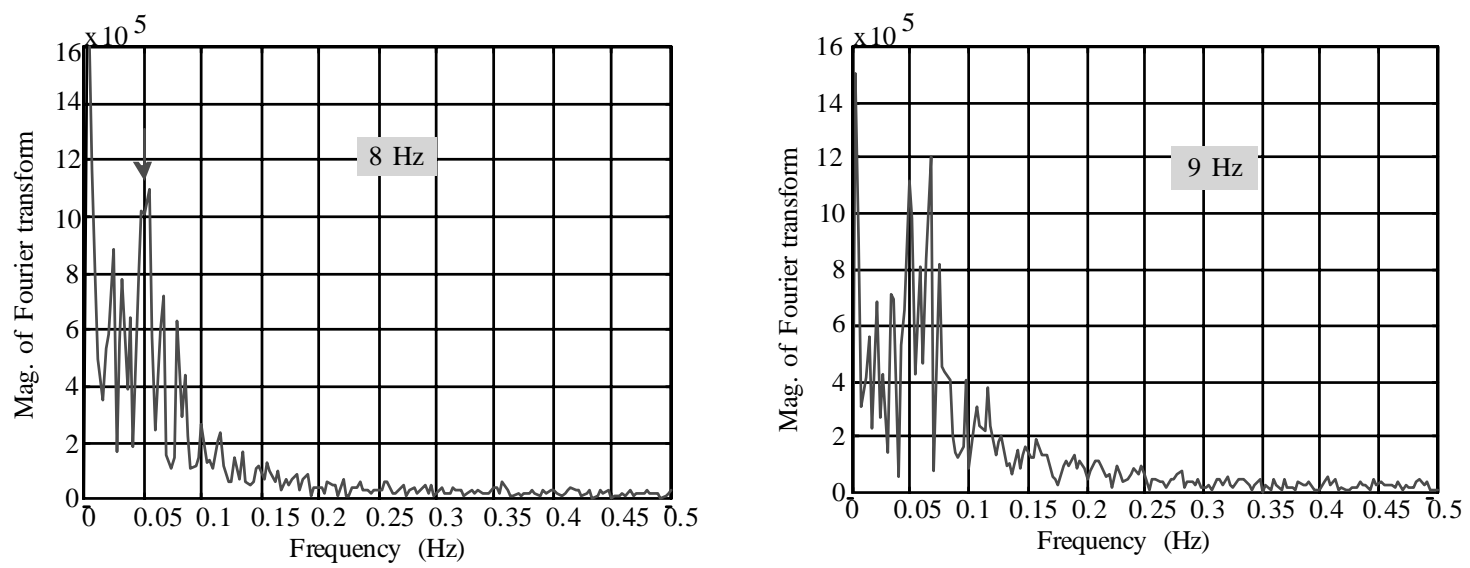

Figure 4: Power spectra for tests done with oscillating 8 and $9 \mathrm{~Hz}$ zoomed between 0 and $0.5 \mathrm{~Hz}$.

The next protocol was designed with a purpose to make another "slow" study, namely to record monocular visual evoked responses [29]. In this protocol, full-field pattern stimulus was applied also. The same checkerboard pattern was reversed at $9 \mathrm{~Hz}$ during $10 \mathrm{~s}$ and the next $10 \mathrm{~s}$ were rest period. During one run, three measurements were done. In the first run, the subject was sitting in front of the pattern with both eyes opened. In the second run, the subject had the right eye covered with a thick black band and in the third run, the subject had the left eye covered with a band. The specific thing about this protocol is that optodes were placed over the left area of the visual cortex only which has to be reflected in the acquired data. In unilateral visual stimulation the effect should be larger in regions of the brain contralateral to the active eye. Therefore, the highest power has to be expressed at the rest/stimulation frequency which is $\sim 0.5 \mathrm{~Hz}$.

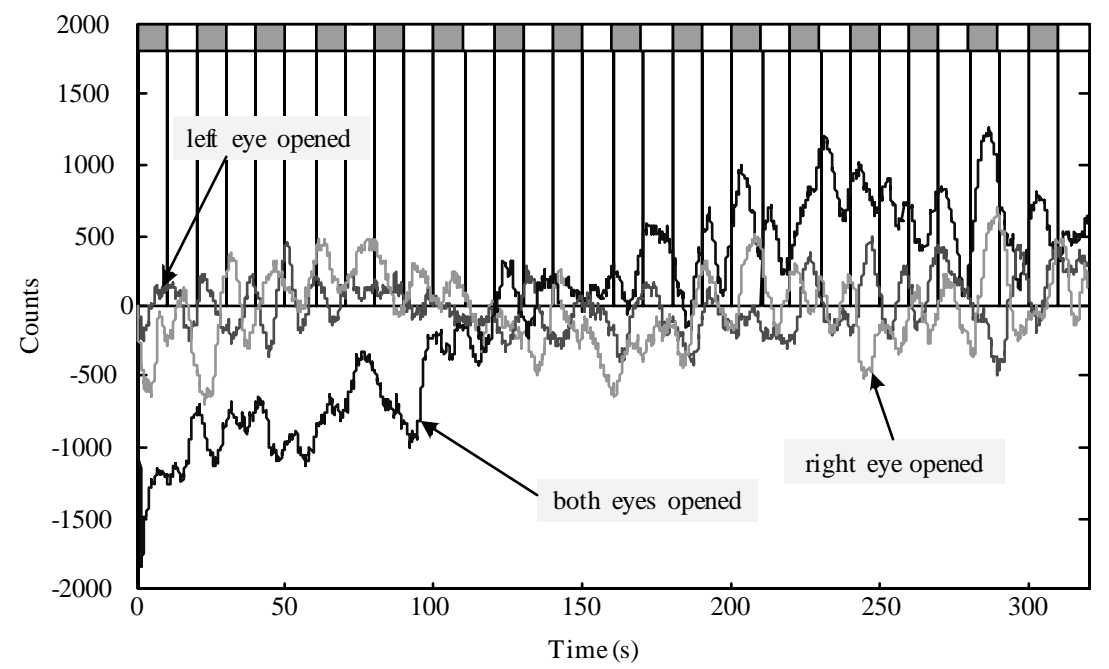

Figure 5: Slow response measured during $320 \mathrm{~s}$ : $10 \mathrm{~s}$ of $9 \mathrm{~Hz}$ pattern reversal marked with dark squares on the top of the figure and $10 \mathrm{~s}$ rest period marked with white squares on the top of the figure.

Figure 5 shows low pass filtered data obtained during these three runs. After low pass filtering, using Butterworth filter, FFT was applied for these three runs. Figure 6 shows three power spectra for each run of the test. 

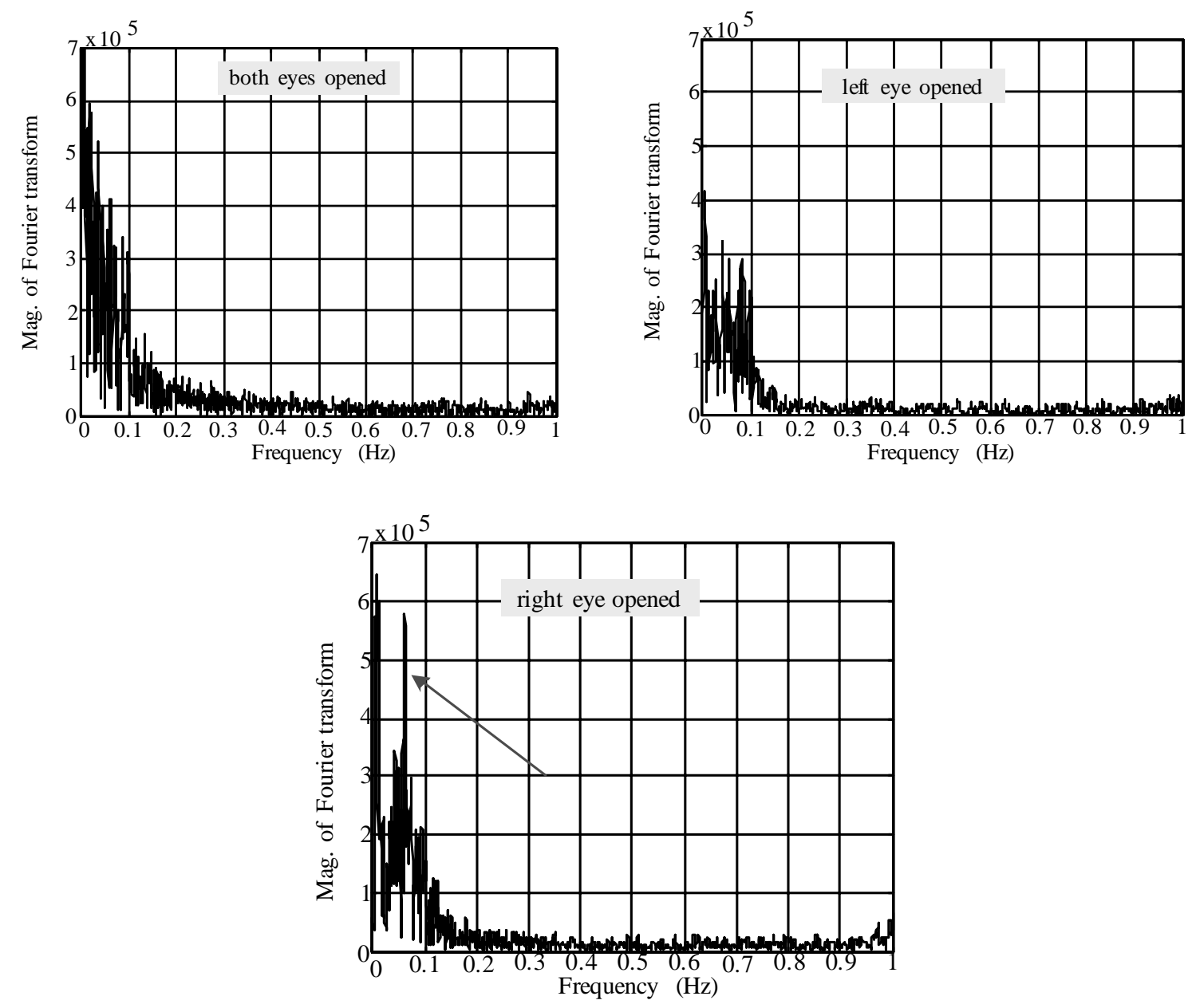

Figure 6: Fourier analysis of data obtained during tests performed during $320 \mathrm{~s}$ with $10 \mathrm{~s}$ of $9 \mathrm{~Hz}$ pattern reversal and $10 \mathrm{~s}$ rest time as shown on Figure 4.

Power spectra of the tests, where both eyes were open and were only left one was opened showed an obvious decrease in response on the lower frequencies in the second case. The power of the record from contralateral eye to the optode showed a nice resonance around the expected frequency $0.05 \mathrm{~Hz}$. If the response from one eye is abnormal but the response of the other eye is normal, the response to binocular stimulation usually is normal.

\subsection{Acquiring fast optical response}

Due to studies done previously, fast optical response is $0.05 \%$ of the total optical signal recorded in the activated brain area during motor task. Taking into consideration photon level during measurement, number of sweeps required for averaging will be 400 . This means that every 400 sweeps we may register one NAS. Data recorded with multichannel scaler with a sampling rate $2 \mathrm{kHz}$ are containing also other biological noise and were processed off-line. First, they were lowpass filtered in both directions with the $2^{\text {nd }}$ order Butterworth filter. Afterwards, the data were bandpass filtered using Elliptic filter from 1 to $10 \mathrm{~Hz}$ to extract noise due to respiration $(0.3 \mathrm{~Hz})$, heartbeat (approximately $1 \mathrm{~Hz})$, Mayer wave $(0.12 \mathrm{~Hz})$, and also noise on higher frequencies. Below are presented data from three tests done with different protocols. 
In the first protocol, the stimulation pattern was reversing every $500 \mathrm{~ms}$ for 400 sweeps. There were two runs done: one with opened eyes and another one with eyes closed. (see Figure 7).

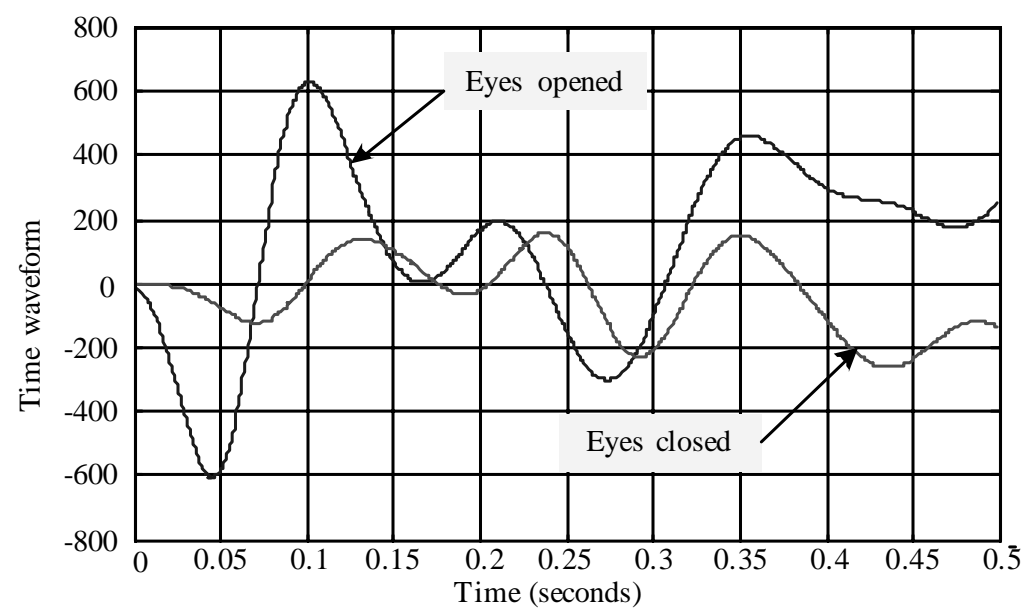

Figure 7: The data acquired during first "fast" study protocol and lowpass filtered.

Data acquired with opened eyes showed a clear peak at $100 \mathrm{~ms}$ after lowpass filtering. A state of eyes-closed was not considered as a "zero" state because of the Alpha band activity during the rest period [30]. Therefore, it is not exactly smooth and can't be considered as a reference signal. The next protocol contains a built-in rest period after every 20 sweeps done for the next 20 sweeps. A rest period causes a "reset" effect for processes occurring in the visual cortex and can "boost" expected fast response. In other words, the improvement made to the protocol has the purpose of increasing the amplitude of neural firing in the visual cortex.
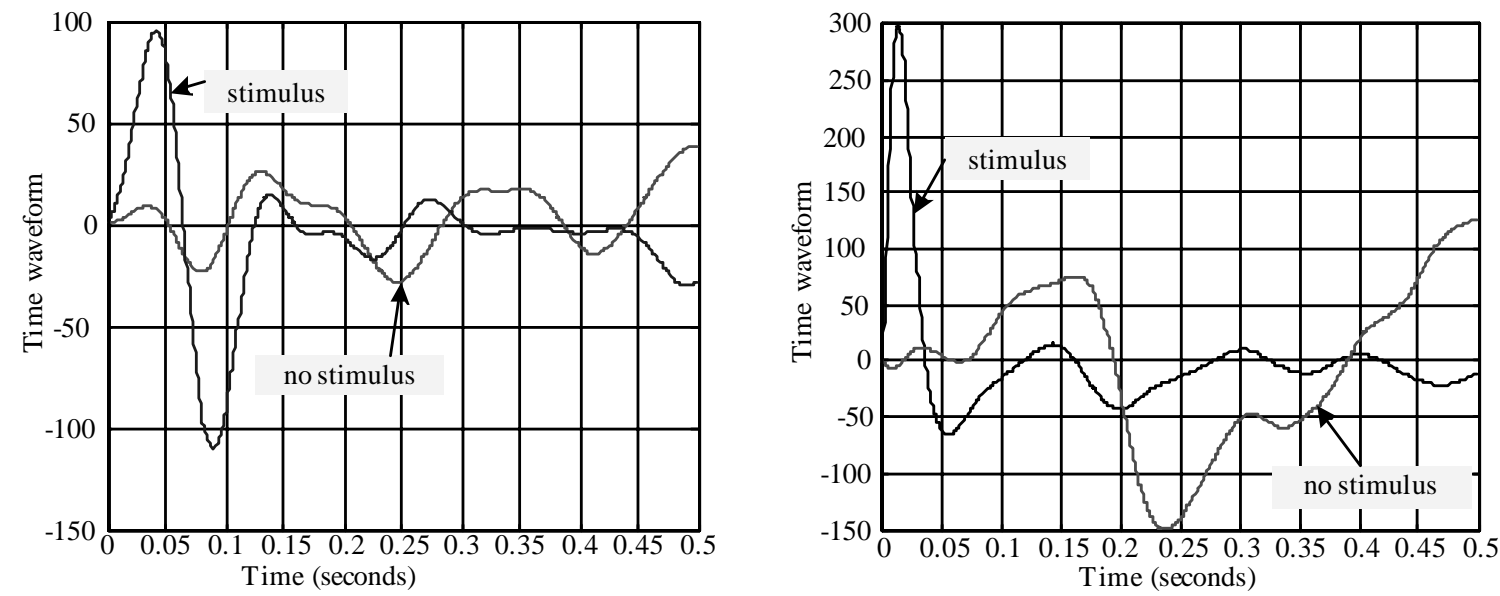

Figure 8: The data acquired during second protocol with built-in rest period from different subjects.

Figure 8 showed the data bandpass filtered from 1 to $10 \mathrm{~Hz}$, which showed usually a peak at $\sim 50 \mathrm{~ms}$ from the on set of the pattern and in the last case it showed a peak at less than $50 \mathrm{~ms}$. The recorded activity has a time course that is consistent with the expected latency of the neural activity since the optode was situated half way closer to the inion as electrode O1. To verify the obtained data it was decided that the latency of the neural activity in the last protocol should be verified using EEG technique. This technique was chosen because of good temporal resolution. 


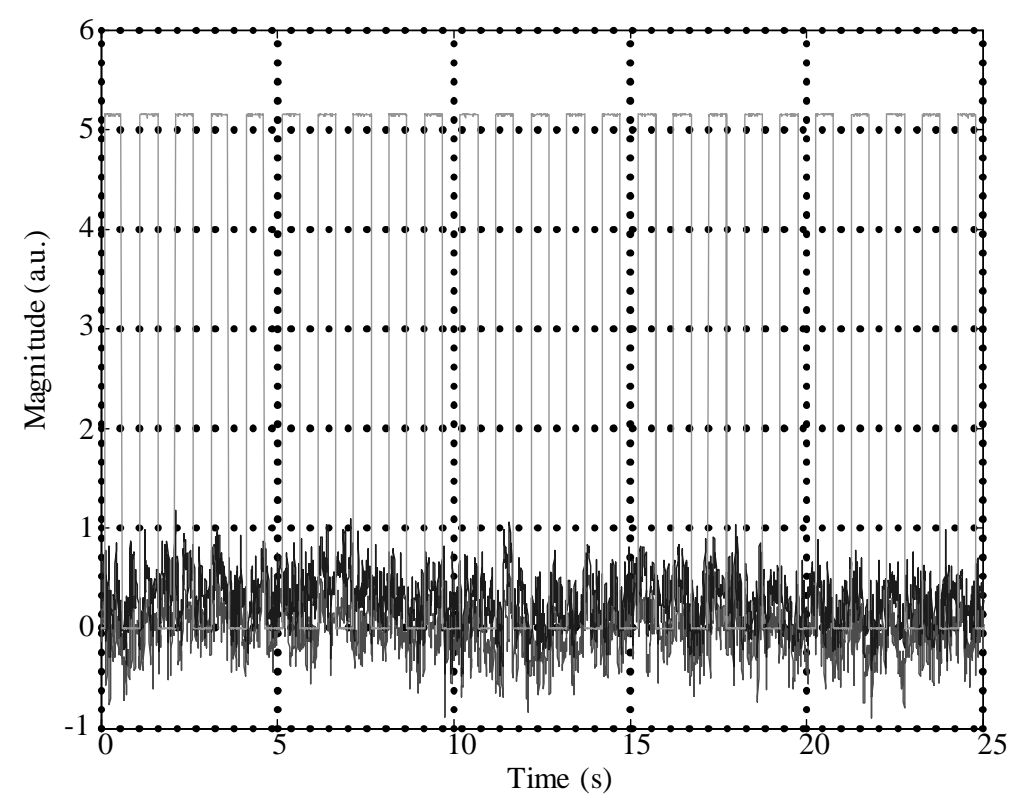

Figure 9: The EEG data acquired during first "fast" study protocol: real time acquisition.
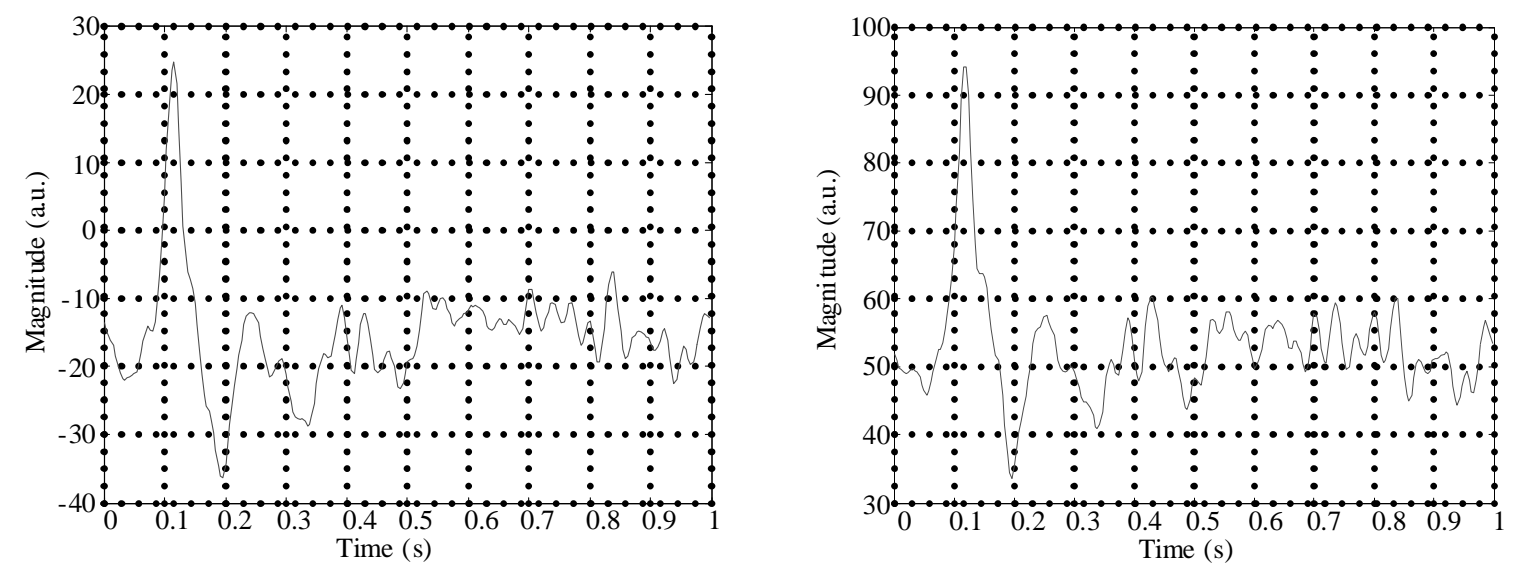

Figure 10: The EEG data acquired during first "fast" study protocol: averaged data from O1 (left) and averaged data from O2 (right).

The EEG recording was done using electrodes placed in the $\mathrm{O} 1$ and $\mathrm{O} 2$ area of the visual cortex [24]. After 240 averages, the acquired signal showed clear peaks from both hemispheres with the latency $\sim 125 \mathrm{~ms}$ (see Figure 9). Normal pattern reversal visual evoked potential showed three major peaks: N75, P100, N145 (see Figure 10). The normal visual evoked potential elicited by pattern reversal stimulation has a number of potential variations. Some of the variability is depending on the patient age, position of the recording electrodes, type of fixation, etc.

\section{CONCLUSIONS}

Various acquisition methods available at present for non-invasive brain analysis are very application-dependent. Only a few techniques can be considered for non-clinical applications with sufficient time-resolution to provide quality measurements on multi-time scales. NIRS and EEG techniques considered here supplement each other and could be 
applied to obtain knowledge in brain processes simultaneously. A time-resolved photon counting technique proved to be a reliable tool in cost-effective studies. The advantages of the presented photon-counting system lie in its exceptional high time resolution even in comparison to EEG system, which gives the possibility to analyse timing of the cortical activities. A disadvantages of the described system are its dark room dependence because of the detector sensitivity and isolation of the subject from any external light influences. Another disadvantage is that time-resolved technique cannot provide knowledge about photon pathlengths through the tissue, which make its application narrower in terms of imaging. Nonetheless, the system acquired clearly hemodynamic changes in the visual cortex of the human brain on the - s time scale and reported measurements on the $-\mathrm{ms}$ timescale. Slow changes in the visual cortex acquired using one wavelength reflect $\mathrm{Hb}$ absorption changes during visual stimulation. Test done for monocular stimulation showed resonance peak response on the stimulation frequency of the contralateral eye to optodes placement as it was predicted. In other words, slow changes in the human brain could be reliably studied using the developed optical technique. On the fast time scale the developed protocols allow the excitation of neural activity in the visual cortex. The data acquired with the optical system gave a rather noisy response. Therefore, off-line signal processing is very important to analyse these data. Comparison of data obtained with optical and EEG system on the ms scale gives tentative evidence that the chosen protocol is functioning well. Result obtained with optical data showed also that it is important to build a rest period into the stimulation protocol, because it increases amplitude of the response. In summary a photon counting technique has been demonstrated capable of measuring optical correlates of brain function both on long and short time scales. The system offers a novel approach for analysing brain function in a multi-modal fashion if used in conjunction with the EEG. Future work will focus on more robust acquisition of the fast optical responses associated with neural firing in the cortex.

\section{ACKNOWLEDGEMENTS}

The principal author would like to acknowledge EMBARK Initiative for funding this project.

\section{REFERENCES}

1. G.N. Martin, Human neuropsychology, Prentice Hall Europe, pp.7-36, 1999.

2. A.W. Toga, J.C. Mazziotta, Brain Mapping The Systems, Academic press, pp. 267-278, 2000.

3. P.L. Madsen, N.H. Secher, "Near-infrared oxymetry of the brain", Progress in Neurobiology, 58, pp. 541-560, 1999.

4. Ch.E. Connor, A. Pasupathy, "Shape representation in ventral pathway visual cortex", International Congress Series, 1269, pp. 23-25, 2004.

5. S.A. Huettel, M.J. McKeown, A.W. Song, S. Hart, D.D. Spencer, T. Allison, G. McCarthy, "Linking hemodynamic and electrophysiological measures of brain activity: evidence from functional MRI and intracranial field potentials", Cerebral Cortex, 14, pp. 165-173, 2004.

6. K. Uludag, D.J. Dubowitz, E.J. Yoder, K. Restom, T.T. Liu, R.B. Buxton, "Coupling of cerebral blood flow and oxygen consumption during physiological activation and deactivation measured with fMRI", NeuroImage, 23, pp.148-155, 2004.

7. M. Volgushev, J. Pernberg, U.T. Eysel, "Response selectivity and $\gamma$-frequency fluctuations of the membrane potential in visual cortical neurons", Neurocomputing, 58-60, pp. 957-963, 2004.

8. R.S. Menon, "Imaging function in the working brain with fMRI", Current opinion in Neurobiology, 11, pp. 630-636, 2001.

9. M.L. Schroeter, M.M. Buecheler, K. Mueller, K. Uludag, H. Obrig, G. Lohmann, M. Tittgemeyer, A. Villringer, D.Y. von Cramon, "Towards a standard analysis for functional near-infrared imaging", Neurolmage, 21, pp. 283290, 2004.

10. G. Jasdzewski, G. Strangman, J. Wagner, K.K. Kwong, R.A. Poldrack, D.A. Boas, "Differences in the hemodynamic response to event-related motor and visual paradigms as measured by near-infrared spectroscopy", NeuroImage, in press, 2003.

11. G. Gratton, M. Fabiani, "The event-related optical signal (EROS) in visual cortex: Replicability, consistency, localisation, and resolution", Psychophysiology, 40, pp. 561-571, 2003.

12. M. Wolf, U. Wolf, J.H. Choi, V. Toronov, L.A. Paunescu, A. Michalos, E. Gratton, "Fast cerebral functional signal in the 100-ms range detected in the visual cortex by frequency-domain near-infrared spectrophotometry", Psychophysiology, 40, pp. 521-528, 2003. 
13. S. Lebid, R. O’Neill, C. Markham, T. Ward, S. Coyle, "Functional Brain Signals: A photon counting system for brain activity monitoring”, IEE Conference Proceedings of The Irish Signals and Systems Conference 2004, Queen's University, Belfast/NI, pp. 469-474, 2004.

14. S. Lebid, R. O’Neill, C. Markham, T. Ward, S. Coyle, “Acquiring functional brain signals with photon counting systems: A time domain approach for pattern reversal steady-state visual evoked potentials at multiple time scales", European Workshop on Optical Fibre Sensors '04, Santander/Spain, invited poster, 2004.

15. J.A. Martin-Pereda, "Learning from the living bodies: New principles and techniques for photonic sensing, invited paper”, European Workshop on Optical Fibre Sensors '04, Santander/Spain, pp. 1-8, 2004.

16. Y. Hoshi, "Functional near-infrared optical imaging: Utility and limitations in human brain mapping", Psychophysiology, 40, pp. 511-520, 2003.

17. A. Franceschini, V. Toronov, M.E. Filliaci, E. Gratton, S. Fantini, "On-line optical imaging of the human brain with 160-ms temporal resolution", Optics express, 6, 3, pp.49-57, 2000.

18. S. Coyle, T. Ward, C. Markham, G. McDarby, "On the suitability of Near-Infrared Systems for Next Generation Brain Computer Interfaces”, Physiological Measurement Special Issue: World Congress on Medical Physics and Biomedical Engineering 2003, 25, pp. 815-822, 2004.

19. H. Obrig, H. Israel, M. Kohl-Bareis, K. Uludag, R. Wenzel, B. Müller, G. Arnold, A. Villinger, "Habituation of the visually evoked potential and its vascular response: implications for neurovascular coupling in the healthy adult". NeuroImage, 17, pp. 1-18, 2002.

20. M.A. Franceschini, D.A. Boas, "Noninvasive measurement of neuronal activity with near-infrared optical imaging", NeuroImage, in press, 2004.

21. G. Gratton, A. Sarno, E. Maclin, P.M. Corballis, M. Fabiani, "Toward non-invasive 3-D imaging of the time course of cortical activity: Investigation of the depth of the event-related optical signal", NeuroImage, 11, pp.491-504, 2000 .

22. D.M. Rector, R.F. Rogers, J.S. Schwaber, R.M. Harper, J.S. George. „Scattered-light imaging in vivo tracks fast and slow processes of neurophysiological activation", NeuroImage, 14(5), pp. 977-94, 2001.

23. R.A. Stepnoski, A. LaPorta, F. Raccuia-Behling, G.E. Blonder, R.E. Slusher, D. Kleinfeld, "Noninvasive detection of changes in membrane potential in cultured neurons by light scattering", Proceeding of National Academy of Sciences USA, 88, pp. 9382-9386, 1991.

24. U. Herwig, P. Satrapi, C. Schönfeldt-Lecuona, "Using the International 10-20 EEG System for Positioning of Transcranial Magnetic Stimulation”, Brain Topography, 24, vol. 16, no. 2, pp. 95-99, 2003.

25. E. Okada, M. Firbank, M. Schweiger, S.R. Arridge, M. Cope, D.T. Delpy, "Theoretical and experimental investigations of near-infrared light propagation in a model of the adult head", Applied Optics, 36, pp. 21-31, 1997.

26. R. W. O'Neill, P. J. M. van der Burgt, D. Dziczek, P. Bowe, S. Chwirot and J. A. Slevin. "Polarization correlation measurements of electron impact excitation of H(2p) at 54.4eV", Physical Review Letters, 80, pp. 1630-1633, 1998.

27. A.G. Hudetz, B.B. Biswal, H. Shen, K.K. Lauer, J.P. Kampine, "Spontaneous fluctuations in cerebral oxygen supply. An introduction”, Adv. Exp. Med. Biol., 454, pp.551-559, 1998.

28. G. Morren, M. Wolf, P. Lemmerling, U. Wolf, J.h. Choi, E. Gratton, L.De Lathauwer, S. Van Huffel, "Detection of fast neuronal signals in the motor cortex from functional near infrared spectroscopy measurements using independent component analysis", Medical \& Biological Engineering \& Computing, 42, pp. 92-99, 2004.

29. K.E. Misulis, T. Fakhoury, Spehlmann's evoked potential primer, Butterworth Heinemann, 2000.

30. M. Moosmann, P. Ritter, I. Krastel, a. Brink, S. Thees, F. Blankenburg, B. Taskin, H. Obrig, A. Villringer, "Correlates of alpha rhythm in functional magnetic resonance imaging and near infrared spectroscopy", NeuroImage, 20, pp. 145-158, 2003. 